\title{
High-resolution ultrasound evaluation in the follow- up of treat to target urate-lowering therapy in gout: an observational study
}

\section{Weijing Zhang}

Nanjing University Medical School Affiliated Nanjing Drum Tower Hospital

\section{Di Zhao}

Nanjing University Medical School Affiliated Nanjing Drum Tower Hospital

Min Wu

Nanjing University Medical School Affiliated Nanjing Drum Tower Hospital

Zhibin Jin ( $\square$ Jin.jzb@icloud.com )

Huayong Zhang

Nanjing University Medical School Affiliated Nanjing Drum Tower Hospital

Research article

Keywords: Gout, Ultrasound, Urate-lowering therapy, Outcome measures

Posted Date: July 15th, 2020

DOI: https://doi.org/10.21203/rs.3.rs-41654/v1

License: (c) (1) This work is licensed under a Creative Commons Attribution 4.0 International License.

Read Full License 
1 High-resolution ultrasound evaluation in the follow-up

2 of treat to target urate-lowering therapy in gout: an

3 observational study

4 Weijing Zhang $\mathrm{PhD}^{1}$, Di Zhao $\mathrm{MD}^{1}$, Min Wu MD ${ }^{1}$, Zhibin Jin $\mathrm{MD}^{1 *}$

5 Huayong Zhang $\mathrm{PhD}^{2 *}$

$6 \quad{ }^{1}$ Department of Ultrasound Medicine, Nanjing Drum Tower Hospital, The

7 Affiliated Hospital of Nanjing University Medical School, No.321

8 Zhongshan Road,210008, Nanjing, Jiangsu, P.R. China

$9 \quad{ }^{2}$ Department of Rheumatology and Immunology, Nanjing Drum Tower

10 Hospital, The Affiliated Hospital of Nanjing University Medical School,

11 No.321 Zhongshan Road,210008, Nanjing, Jiangsu, P.R. China

12 *Correspondence: jin.jzb@icloud.com; ; huayong.zhang@nju.edu.cn ${ }^{2}$

13 Conflicts of Interest and Source of Funding: The authors declare no 14 conflicts of interest. 


\title{
High resolution ultrasound evaluation in follow-up of treat to target urate-lowering therapy in gout: an observational study
}

\author{
Abstract
}

Background: The monitoring of treat-to-target (T2T) urate-lowering therapy (ULT) for gout is crucial for the assessing treatment response. However, evidence is lacking about clinical remission on ultrasound (US). The aim of this study was to observe the changes in three outcome domains (urate deposition, joint inflammation and bone erosion) in patients with ULT within 1 year, evaluate the effect of target treatment and analyse the relationships between clinical factors and US features.

Methods: The elementary lesions of the bilateral knee, ankle and first metatarsophalangeal joints were evaluated by US before and after 3,6 and 12 months of treatment. Urate deposition was assessed by the maximum long and short axis diameters of the tophi and dichotomous data of the double-contour (DC) sign and aggregates. After each follow-up, the most obvious lesions were selected for repeated observation. The effective clearance rates of these three signs in different time groups were compared. A Global OMERACT-EULAR Synovitis Score (GLOESS) was calculated for these three paired joints to observe the remission and recurrence of inflammation. Bone destruction was scored at each time point. The correlation between serum uric acid (sUA) levels and tophi size changes was analysed. 
1 Results: This cohort contained 79 patients. The long and short axis

2 diameters of tophi showed a different descending tendency. The decrease

3 of sUA levels correlated with the decrease of long axis values, but not with

4 the short. For tophi, there was no significant difference in the clearance rate

5 between different time groups, while for DC sign and aggregates,

6 significant differences were found by paired comparison. The GLOESS

7 was significantly lower after 6 months of therapy. Bone erosion had not

8 been improved after 1 year of ULT.

9 Conclusion: The decrease in sUA levels was not completely parallel to the

10 decrease in tophi size. ULT with different intensities should be formulated

11 according to different crystal deposition conditions under US assessment.

12 Subclinical inflammation was gradually controlled after 6 months of 13 therapy and can be sensitively observed by US. Joint damage was relatively 14 stable within 12 months of ULT.

15 Trial registration: ChiCTR1800015043

Keywords: Gout, Ultrasound, Urate-lowering therapy, Outcome measures Introduction without treatment will eventually have a reduced quality of life, decreased capacity to work and increased risk of death due to joint damage, kidney impairment and an increase in cardiovascular events ${ }^{[1]}$. Timely and effective treatment and monitoring may control disease progression, avoid 
1 adverse events and ultimately reduce mortality. Urate-lowering therapy

2 (ULT), as an effective gout management strategy, has been widely

3 recognized as crucial in delaying the disease progression and reducing

4 damage to the functions of certain viscera. Unfortunately, direct best-

5 quality evidence is not available for the treat-to-target (T2T) approach.

6 Different therapeutic targets may depend on the severity of the disease and

7 the stage of treatment ${ }^{[2]}$. There is an overall agreement to consider serum

8 uric acid(sUA) levels below $6 \mathrm{mg} / \mathrm{dL}(360 \mu \mathrm{mol} / \mathrm{L})$ as the minimum target

9 and below $5 \mathrm{mg} / \mathrm{dL}(300 \mu \mathrm{mol} / \mathrm{L})$ for severe gout. These therapeutic targets

10 have been endorsed by both the 2016 European League Against

11 Rheumatism (EULAR) ${ }^{[3]}$ and 2012 American College of Rheumatology (ACR) ${ }^{[4]}$ recommendations. However, the sUA level is affected by many factors and changes over time, so it cannot truly reflect the whole-body urate burden. In addition, it takes several months for monosodium urate (MSU) crystals deposited in the joint capsule to dissolve with normal sUA, and the dissolution rate is correlated with other factors, such as disease duration $^{[5]}$. To date, there are no remission criteria established for gout ${ }^{[6]}$. Considering that and the endpoints of ULT vary greatly, imaging evidence of MSU is feasible for the guidance of clinical medication because the disappearance or decrease of MSU in imaging may reflect the effectiveness of treatment to some extent.

To date, the impact of urate-lowering drugs on the changes in imaging 
1 during the T2T approach is not very clear. The Outcome Measures in

2 Rheumatology (OMERACT) gout working group identified three key

3 domains for imaging modalities to assess: urate burden, joint inflammation

4 and structural damage ${ }^{[7]}$. Different modalities have superiority and

5 insufficiency for different domains ${ }^{[8]}$. Dual energy computed tomography

6 (DECT) has the potential for urate deposition and bone damage

7 observation but is not recommended for joint inflammation assessment ${ }^{[9,}$

$8{ }^{10]}$. MRI is good at showing bone erosion and synovitis, however, for most

9 clinically apparent tophi, measurement using MRI has little advantage over

10 less expensive, safer and faster methods of physical measurement ${ }^{[8]}$.

11 Compared with other imaging modalities, ultrasound (US) has the 12 highest performance for all three domains ${ }^{[7]}$, and is expected to help 13 clinicians evaluate the intensity of ULT, increase the treatment compliance 14 of patients, and guide them to achieve more ideal clinical and imaging 15 outcomes.

Few studies have been reported on high-frequency US evaluation in 17 the follow-up of ULT. Previous studies suggested that US had a high specificity and positive predictive value in the diagnosis of gout ${ }^{[11]}$, and 19 played a certain role in the monitoring of therapy ${ }^{[12-14]}$. However, most of these studies focused on the sensitivity of US features (such as doublecontour (DC)sign and tophi) to ULT ${ }^{[14,15]}$ and evidence is lacking about the specific changes of those features during different treatment periods. 
1 Meanwhile, MSU deposition is often associated with joint inflammation

2 and structural damage, but there are few reports on the response to

3 treatment of these two domains by US.

4 The primary objective of this study was to describe the progression of

5 US features from those three aspects before and 3, 6, and12 months after

6 T2T therapy. Other objectives included identifying whether the observed

7 changes in imaging in response to treatment are associated with changes in

8 clinical data and providing a perspective on the role of US imaging for

9 measuring treatment response in patients with gout.

\section{Methods}

\section{Study design}

We designed a 1-year prospective, observational US study involving gouty patients initiated to receive regular ULT. All patients were treated according to clinical routine and no intervention was conducted during the whole treatment. Patients could also take colchicine, NSAIDs or corticosteroids if needed. US assessment was performed at baseline and at 3, 6 and 12 months after ULT initiation. The study was approved by the Ethics Committee of Nanjing Drum Tower Hospital (No.2018-013-01) and all the enrolled patients signed an informed consent form.

\section{Patients}

Participants were selected consecutively form outpatients in our hospital between July 2017 and March 2018. Patients were eligible if they were 
1 positive for MSU crystals in synovial fluid. The exclusion criteria included

2 the following: (1)age $<18$ or $>80$ years old; (2)presence of other rheumatic

3 diseases; (3) administration of regular ULT or corticosteroid injection

4 within the previous 3 months; and (4) creatinine clearance of $<20 \mathrm{ml} / \mathrm{min}$

5 or severe liver function lesions (liver enzymes 3 times the upper limit of

6 normal). Participants who took medicine irregularly and had no follow-up

7 records were also excluded from the final statistical analysis. To evaluate

8 medication adherence, the proportion of days covered (PDC) was used, and

$9 \quad$ PDC $\geq 80 \%$ was considered adherent ${ }^{[16]}$. At baseline, all patients underwent

10 a detailed clinical evaluation, including disease history (disease duration,

11 number of gout flares, duration of attack, comorbidities, etc.),

12 demographics and clinical characteristics (age, sex, BMI, tender joint count

13 (TJC) and swollen joint count (SJC)), and laboratory testing (sUA levels,

14 serum creatinine levels, serum urea nitrogen levels) (table 1). Some of the

15 clinical data and laboratory testing were assessed at 3,6 and 12 months 16 after treatment.

17 Primary and secondary outcomes

18 The primary outcome was the reduction or disappearance of specific US

19 features (tophi, DC sign and aggregates) after 12 months of ULT. The 20 secondary outcome was that two measurements of sUA stayed below $21360 \mu \mathrm{mm} / \mathrm{L}$ at least six months apart. 
1 US examinations were performed by a single sonographer experienced in

2 musculoskeletal US, who was unacquainted with the clinical data. The

3 examinations were conducted using a Toshiba Aplio500 scanner (Toshiba,

4 Tokyo, Japan) with a 4-12 MHz linear array transducer. The power Doppler

5 (PD) frequency was 4.4-6.1 MHz. A medium wall filter was used and the

6 gain was adjusted until the background noise was suppressed. The joints in

7 which gout lesions were detected included the bilateral knee, ankle and first

8 metatarsophalangeal (MTP) joints of each patient. Suprapatellar

9 longitudinal and transverse planes as well as medial and lateral longitudinal

10 planes were scanned with the knee in the $30^{\circ}$ flexion position, while the

11 femoral cartilage was examined in the axial plane with full knee flexion.

12 To scan the ankle, the patients were asked to place their feet flat on the 13 examination bed. The anterior, medial and lateral regions were scanned 14 sequentially along the longitudinal and transverse axes. The first MTP joint 15 was examined from the dorsal, plantar and medial views in the longitudinal 16 and transverse planes according to the scanning technique described in the 17 European guidelines for musculoskeletal ultrasound ${ }^{[17]}$.

18 Urate deposition was represented as tophi, DC sign and aggregates on 19 US, which were defined by recently formed OMERACT consensus-based 20 definitions $^{[18]}$. At baseline, these three items were systematically examined 21 for each joint. The DC sign and aggregates were recorded as dichotomous 22 data (presence or absence) at each visit. For tophi, we selected the most 
1 obvious one (usually in the MTP1 joint for the high detection rate ${ }^{[19]}$ ) as

2 the target at baseline, measured the longest diameter on the longitudinal

3 and transversal axes from the medial side and repeated the measurement at

43,6 , and 12 months of follow-up.

5 PDUS evaluation was performed at each site 4 times. The PDUS

6 assessment consisted of an evaluation of greyscale synovial hyperplasia

7 (SH) and intrasynovial PD signal. Each single component of joint

8 inflammation (SH and PD) was scored separately using a semiquantitative

9 scale of $0-3^{[20]}$. To monitor disease activity, we used the composite PDUS

10 score according to the OMERACT-EULAR PDUS composite

11 semiquantitative scale $(0-3)$ at the joint level ${ }^{[21]}$, which is analogous to the

12 composite scoring system used in rheumatoid arthritis (RA). The Global

13 OMERACT-EULAR Synovitis Score (GLOESS) for these 3 paired joints

14 was then calculated using the sum of the composite PDUS scores for all 15 joints examined ${ }^{[22]}$, ranging from 0-18.

At baseline, the presence of erosion was also recorded with a 0-3 scale

17 for each joint ${ }^{[20]}$ and confirmed on longitudinal and transverse scans. At 18 each follow-up, the same joint with the most obvious bone destruction was

19 selected for reevaluation. All images were saved and stored in the hard 20 drive of the US machine for further analysis.

21 Statistical analysis

22 Statistical analyses were performed using SPSS Version 23.0 (IBM, 
1 Armonk, NY, USA). Normally distributed variables are reported as the

2 mean (SD), and categorical variables are reported as the frequencies.

3 Student's t test was used for quantitative variables, and the chi-square test

4 or Fisher's exact test was used for categorical data. Repeated measurement

5 data of sUA and tophi size were compared using one-way analysis of

6 variance (ANOVA). Correlations between US findings and clinical

7 characteristics were estimated by the Spearman correlation coefficient.

8 Changes in clinical and US inflammatory indicators from baseline to

9 month 12 were investigated by several related samples tests (Friedman's

10 M test). Bone erosion before and after treatment was compared by the

11 Wilcoxon matched-pairs signed rank test. $\mathrm{P}<0.05$ was considered 12 statistically significant.

\section{Intra- and interobserver agreement}

14 The interobserver reliability of the US features of gout (erosion, $\mathrm{SH}$ and

15 PD) and tophi size measurement were calculated by two sonographers with 16 more than 5 years of experience in musculoskeletal US using 30 images 17 from 10 randomly selected patients. Two weeks after the initial assessment, 18 one of the sonographers under blinded conditions re-analysed the images 19 to evaluate the intraobserver reliability. For the measure of tophi size, a 20 discrepancy $>20 \%$ between the two measures of two US operators 21 (interobserver) or between the same operator (intraobserver) was considered different. The concordance for US was estimated as the kappa 
1 coefficient, with $\kappa>0.8$, almost perfect; $0.6-0.8$, good; 0.4-0.6, moderate,

$2 \quad 0.2-0.4$, fair, and $\leq 0.2$, poor.

\section{Results}

4

\section{Clinical characteristics}

A total of 79 individuals with regular ULT were recruited. The patient demographics are shown in table 1. Seventy-five (94.9\%) patients received febuxostat for the treatment, with a mean dose of $35.2 \pm 12.2 \mathrm{mg} / \mathrm{d}$, and another $4(5.1 \%)$ subjects chose benzbromarone, with a mean dose of 39.7 $\pm 14.7 \mathrm{mg} / \mathrm{d}$. The number of patients who completed treatment at months 3 , 6 and 12 were $67(85.9 \%), 64(81 \%)$ and 57 (72.2\%), respectively.

With treatment, the symptoms of the patients were gradually relieved. We found that SJC, TJC, number of gout flares and duration of attack differed at different time points $(\mathrm{P}<0.001)$. The paired comparison results showed that SJC was significantly reduced by 6 months $(\mathrm{P}=0.043)$, while TJC decreased by 3 months $(\mathrm{P}=0.006)$. The number of gout flares decreased significantly by 12 months compared with that at baseline and 3 months $(\mathrm{P}<0.001$ and $\mathrm{P}=0.002$, respectively), and the duration of attack gradually shortened from 3 months $(\mathrm{P} \leq 0.01)$ (table1).

\section{Urate deposition changes in response to ULT}

At baseline, tophi was found in 48 patients (60.8\%), and the mean changes in the long and short axes of diameters are listed in table 2. The DC sign was present in 53 patients $(67.1 \%)$ and hyperechoic aggregates were 
1 detected in 44(55.7\%) participants. The proportion of patients with these

2 three features observed at different time points is shown in table 2 .

3 Interobserver and intraobserver agreement

4 The interobserver agreement for the measure of tophi and US feature

5 scoring were as follows: tophi: 0.45 (95\% CI: -0.124 to 1.024$)$; bone

6 erosion: 0.616 (95\%CI: 0.249 to 0.983$)$; $\mathrm{SH}: 0.853$ (95\%CI: 0.579 to

7 1.127); and PD: 0.902 (95\%CI: 0.716 to 1.088$)$. The intraobserver

8 agreement for all those features were as follows: tophi: 0.633 (95\%CI:

90.004 to 1.262 ); bone erosion: 0.732 (95\%CI: 0.407 to 1.057 ); SH: 0.874

(95\%CI: 0.637 to 1.111 ); and PD: 0.920 (95\%CI: 0.767 to 1.073 ).

11 Changes in sUA levels and tophi size over 12 months

Overall, the level of sUA showed decreasing tendency, and the difference was statistically significant $(\mathrm{F}[2.349,77.530]=25.863, \mathrm{P}<0.001)$. After 6 months of treatment, the level of sUA remained basically stable. Twentyfour patients $(42.1 \%)$ reached the sUA objective $(<360 \mu \mathrm{mol} / \mathrm{l})$ at month 12.

Both the long and short axis diameters of tophi decreased significantly at follow up (for long axis: $\mathrm{F}[1.616,42.003]=7.41, \mathrm{P}=0.003$; for short axis: $\mathrm{F}[2.187,56.855]=10.95, \mathrm{P}<0.001)$. However, the long and short axes of tophi showed different trends. The maximum long axis diameter displayed a significant reduction until month 12 . Compared with that at baseline and month 3 , the long axis diameter measured at month 12 decreased by 0.22 
$1 \mathrm{~cm}(\mathrm{P}=0.018)$ and $0.17 \mathrm{~cm}(\mathrm{P}=0.02)$, respectively. The earliest reduction in

2 the short axis diameter of the tophi was observed as early as month 3 and

3 then gradually declined. The short axis values at months 3,6 and 12

4 dropped by $0.072 \mathrm{~cm}(\mathrm{P}=0.038), 0.111 \mathrm{~cm}(\mathrm{P}=0.003)$ and $0.156 \mathrm{~cm}(\mathrm{P}=0.02)$

5 respectively, compared with the baseline value (figure 1).

6 The changes in sUA levels were positively correlated with the changes

7 in the long axis value of tophi $(\mathrm{r}=0.335, \mathrm{P}=0.043)$; however, no correlation

8 was found between the decrease of sUA level and the short axis value $9 \quad(\mathrm{r}=0.287, \mathrm{P}=0.085)$. There was no significant correlation between disease 10 duration and the reduction in long and short axis diameters.

11 Urate deposition clearance follow-up after ULT

To evaluate the effectiveness of ULT, we introduced the concept of the effective clearance rate, which was defined as the proportion of subjects with DC signs or aggregates from presence to absence or with both the long and short axis diameters of tophi decreasing by $20 \%$ before and after ULT. The efficacy of the treatment for tophi was $5 \%(2 / 40), 12.5 \%(5 / 40)$ and $5.4 \%(2 / 37)$ at months 3,6 and 12 , respectively, and the clearance rate did not differ significantly between the different time groups $\left(\chi^{2}=1.76\right.$, $\mathrm{P}=0.392)$. Throughout the whole study period, 2 patients $(5 \%)$ had their tophi totally dissolved at month 6 . The differences in the effective clearance rate for the DC sign and aggregates among the three follow-up groups were statistically significant (DC sign: $\chi^{2}=21.48, \quad \mathrm{P}<0.001$; 
1 aggregates: $\left.\chi^{2}=7.75, P=0.018\right)$. No patients were observed with the $\mathrm{DC}$ sign

2 disappearing by 3 months of follow-up. However, the DC sign clearance

3 rate significantly improved at month $6(19.1 \%)$ and month $12(34.2 \%)$

$4 \quad(\mathrm{P}<0.001)$. Aggregates disappeared as early as 3 months in $7 \%$ of the

5 patients (3/43). There were no significant between-group differences in the

6 clearance rate at the neighbouring time point; however, the month 12 rate

7 was higher than the month 3 rate $(32.2 \%$ (10/31) vs $7 \%(3 / 43), \mathrm{P}=0.01)$

8 (figure 2).

\section{Disease activity follow-up}

There were a total of 65 patients $(82.3 \%)$ with joint inflammation at baseline. Thirty-four (52.3\%) of them had only one joint involved, with 3 patients $(8.8 \%)$ involved in the knees, $16(47.1 \%)$ in the ankles and 15 (44.1\%) in MTP1, while the rest had multiple joints affected. A reduction trend was observed after the initiation of ULT, and the proportion of patients with detected joint inflammation was $76.1 \%(51 / 67), 70.3 \%(45 / 64)$ and $57.9 \%(33 / 57)$ at months 3,6 and 12, respectively. The GLOESS at each time point was significantly different $\left(\chi^{2}=32.316, \mathrm{P}<0.001\right)$. Paired comparisons suggested that the values at baseline (range 0-11, median 3) were significantly higher than those at 6 months (range $0-10$, median 1 ) $(\mathrm{P}=0.004)$ and 12 months (range $0-6$, median 1) $(\mathrm{P}<0.001)$. That is, inflammation became gradually controlled after 6 months of treatment (figure 3). 
2 Forty-eight patients (60.8\%) had bone erosions, 27 (56.3\%) of whom had

3 only one joint involved, and the others had multiple. The majority of those

4

\section{Bone erosion follow-up} were located at MTP1 followed by the ankle. At baseline, we found that $10.1 \%$ of the patients had grade 1 erosions, and the proportions for grades 2 and 3 were $13.9 \%$ and $36.7 \%$, respectively. At month 12 , the corresponding values for grades 1,2 and 3 were $8.8 \%, 15.8 \%$ and $40.3 \%$, respectively. After 1 year of treatment, the rating increased in 3 patients ( 1 was upgraded from grade 0 to grade 1), while the other 54 subjects had their score remained unchanged, and no rating downgrade was found. There was no significant difference in the US rating of bone erosions before and after 1 year of treatment $(\mathrm{Z}=-1.633, \mathrm{P}=0.102)$ (figure 4).

\section{Discussion}

There is a considerable amount of indirect evidence that T2T treatment for gout is clinically effective in real practice ${ }^{[2]}$. The ultimate goal is to lower sUA levels, which is beneficial for promoting the dissolution of MSU in tissue. Theoretically, effective treatment strategies will sufficiently reduce sUA levels to prevent further crystal formation and dissolve existing urate crystals, thus eliminating the causative agent and effectively 'curing' the disease $^{[23]}$. Therefore, it is necessary to monitor the dissolution of MSU in vivo to better evaluate the efficacy of therapy, and a newly published metaanalysis demonstrated that US offers good diagnostic accuracy with high 
1 specificity that may play an important role in the monitoring of disease ${ }^{[24]}$.

2 We conducted a 12-month follow-up study to evaluate the disappearance

3 of the US features of gout under efficient ULT. Three elementary lesions

4 of urate crystal deposits on US: DC sign, tophi and aggregates ${ }^{[18]}$ were

5 mainly observed.

6 Clearance of crystal deposits

7 Patients with advanced gout usually present with large tophaceous deposits.

8 Many challenges still exist in quantitating them on US. We found that the

9 short axis diameter was significantly decreased by month 3 and then

10 maintained a steady decline. The long axis diameter fell slowly, and the

11 difference was statistically significant until month 12 compared with that 12 at baseline, which was not completely consistent with the short axis 13 diameter. This is possibly due to the smaller base of the short axis diameter, 14 and the acoustic shadow of the tophi may also affect the measurement 15 accuracy. Therefore, the long axis diameter rather than the short axis diameter is more suitable to reflect tophi size changes considering system 17 bias.

In our study, the mean size of the tophi shrank; however, some patients had an enlarged tophus during ULT. A previous study has demonstrated that some tophi enlarge before beginning to resolve ${ }^{[25]}$. Such enlargement represents tophi remodelling during the early phases of resolution, which may result in reduction slowing ${ }^{[26]}$.The sUA level is the most important 
1 indicator in monitoring disease. There is ample evidence of the effect of

2 sUA normalization on tophi resolution ${ }^{[15,27,28]}$. However, the concrete

3 relationship between the sUA level and MSU clearance rate is unclear. The

4 present study showed that the sUA level fell sharply by month 3 and then

5 decreased moderately. This conclusion agrees with a previous report that

6 sUA levels fell significantly from baseline through the 4th month of

7 follow-up and plateaued thereafter ${ }^{[29]}$. Overall, our findings indicate that

8 the changes in sUA levels were not exactly parallel to the tophi diameter

9 changes. At the end of 12 months, there were still 13 patients (54.2\%)

10 achieving therapeutic goals observed with tophi. Therefore, we suggest that

11 continuous monitoring by US is needed even for those who have achieved

12 clinical remission until the tophi completely vanishes.

Our results showed that the dissolution rates of those three lesions were different. The clearance rate of tophi did not differ significantly

15 between the different time groups. As mentioned above, the crystal structure was stable and hard to be dissolved. For the DC sign, Das et al ${ }^{[29]}$ demonstrated that it disappeared mostly by 6 months. Peiteado et al ${ }^{[30]}$ suggested that it was probably much easier to clear cartilage urate deposits than tophi. These results are consistent with our findings. Compared with tophi, a lighter MSU load within the DC sign and disappearance seemed to appear earlier. Among the three signs, aggregates showed the fastest

22 clearance at as early as 3 months of treatment, indicating that under the 
1 same treatment conditions, the curative effect of aggregates is better than

2 that of DC sign and tophi. The difference may be relevant to critical size

3 and time. The smaller the critical size is with a short deposition time, the

4 faster the clean-up and vice versa. These results support the idea that

5 different therapeutic intensities are demanded according to different US-

6 detectable deposits, and intense treatment is needed for patients with

7 tophaceous gout. disappearance time of urate crystals from synovial fluid was correlated

11 with the duration of gout, and a longer time was required in those patients

12 with a longer duration. However, Ebstein et al ${ }^{[15]}$ considered that disease 13 duration was not associated with the modification of DC sign and tophi. In 14 this study, we found no significant correlation between the course of 15 disease and the changes in both the long axis and short axis diameters of tophi, in part because tophi solubility can be affected by variable factors,

17 related to the drug used and the degree of sUA level decrease. Moreover, tophi represents a complex structure with inflammatory tissue located 19 adjacent to the central core of the MSU crystal ${ }^{[31]}$. The heterogeneity itself 20 and the individual variation may also be relevant.

\section{Inflammation}

Previous studies have confirmed that PDUS is able to globally measure the 
1 perfusion changes with high sensitivity in other inflammatory arthritis

2 conditions $^{[32-34]}$. To the best of our knowledge, few studies have reported

3 disease activity follow-up by US in gout to date. It is unclear whether

4 additional value to clinical examination can be provided by PDUS in

5 establishing true gout remission. A previous MRI study showed that ULT

6 with febuxostat improved MRI synovitis and reduced gout flares in

7 subjects with early gout over a 2-year period ${ }^{[35]}$. Another study

8 demonstrated that a Doppler signal at two years persisted in a high 9 percentage of patients $(72.7 \%)$, despite an obvious clinical improvement ${ }^{[13]}$.

10 The present study evaluated the reduction trend of GLOESS and found that

11 inflammation became gradually controlled at 6 months of treatment. In the

12 early weeks and months of treatment, patients using antihyperuricemic

13 drugs usually experience more frequent gout attacks, and then the

14 incidence is reduced afterwards ${ }^{[36]}$. After 12 months of treatment, although

15 most of the patients were in the interictal stage with clinical indicators

16 including SJC and TJC, the number of flares and duration of attack were

17 significantly reduced. The presence of US synovitis in one or more joints

18 was still observed in $56.9 \%$ of subjects. This low-grade inflammation in

19 the joint might be associated with the deposition of tophi, which

20 contributes to a cycle of chronic inflammation, attempted resolution, and

21 tissue remodelling ${ }^{[31]}$. These results support the hypothesis that the

22 disappearance of a Doppler signal requires for the entire dissolution of 
1 MSU deposits. Furthermore, the subclinical activity of gout is often

2 ignored by patients for they have no symptoms, resulting in the interruption

3 of treatment thereafter. Thus, it is necessary to monitor inflammation by

4 PDUS in target areas until no PD signal is found in follow-up visits. Further

5 studies are needed to elucidate the predictive value of the underlying

6 inflammation observed by US and the feasibility of introducing a Doppler

7 signal as an outcome measure in clinical practice.

8 Bone erosion

9 The US technique was found to be superior to conventional radiographs in 10 evaluating small bone changes ${ }^{[37]}$. According to experienced sonographers, 11 the inter- and intraobserver reliability of bone erosion on US ranged from 12 good to excellent ${ }^{[38]}$. However, a longitudinal analysis of bone erosions in 13 response to treatment in gouty patients by US is lacking. A previous 14 observational study evaluating radiographic joint damage in longstanding 15 gout patients showed that most of them had stable radiographic damage associated with progressive radiographic damage ${ }^{[39]}$.

8 Consistent with the findings of the previous study, we observed that no significant change was found in the US score of bone erosions after 1-year of treatment. This result was expected, mainly because the most powerful factor, tophi ${ }^{[40,41]}$, contributes to progressive bone erosion by promoting osteoclast activation $^{[42]}$ and reducing osteoblast viability and function ${ }^{[43]}$. 
1 Another possible explanation is sustained synovitis, such as RA. Wu et al ${ }^{[41]}$

2 conducted a logistic regression analysis and showed that bone sites affected

3 by erosion were 1.87 -fold more likely to lie adjacent to regions of synovial

4 hypertrophy than not. Synovitis stimulated by long-term inflammation

5 rather than acute inflammation is a risk factor for bone erosion. So far, it is

6 unknown whether gouty erosions reliably repair after effective ULT. A

7 small case series demonstrated improvement in radiographic bone damage

8 scores with profound urate lowering in pegloticase responders with severe

9 gout $^{[44]}$. In contrast, no erosion regression occurred in our cohort, at least

10 during the 1-year observational period. The difference may be due to the

11 intensity of ULT. Compared with first-line antihyperuricemic drugs,

12 pegloticase therapy leads to a profound reduction in sUA levels that rapidly

13 relieves patients. This finding could be supported by the hypothesis that

14 the more intensive treatment ${ }^{[45]}$ is and the longer the duration of sustained

15 remission is, the greater the likelihood of radiographic erosion repair.

16 Furthermore, to achieve the prevention or healing of bone erosion, early

17 diagnosis and treatment would also be important, while US may provide a

18 strong imaging basis for timing selection.

19 Limitations

20 This study has some limitations. First, it is difficult to accurately measure

21 the size of tophi due to heterogeneity and irregular shape. A self-control

22 approach with fixed sections and sites was employed to reduce the bias. 
1 However, since the measurement was taken only from the two-dimensional

2 plane, future investigation of three-dimensional ultrasonography is needed

3 to possibly provide accurate volume measurements for tophus. Second,

4 there are limits to observing the intraarticular damage due to the bone

5 cortex resistance of penetration of the US beam. US can identify only

6 erosions and cartilage damage compared with other modalities ${ }^{[7]}$. It is

7 practical to assess treatment response with its advantages of cost,

8 availability, and safety ${ }^{[46]}$. Furthermore, at the end of 12 months, most

9 patients did not reach sUA levels $(<360 \mu \mathrm{mol} / \mathrm{l})$. The influential factor may

10 be the relatively low dose of medication (a starting dose of $40 \mathrm{mg} / \mathrm{d}$, then

11 increased to $80 \mathrm{mg} / \mathrm{d})^{[47]}$ received by our subjects, which could postpone

12 the achievement of the sUA target. In fact, we chose to perform a US

13 assessment in a real-life condition with no intervention conducted, to better

14 determine the role of US follow-up in clinical practice. Finally, we did not

15 consider diet and lifestyle factors, and the influence on US changes remains

16 unclear. Future long-term studies with larger cohorts are needed to provide

17 more comprehensive explanations.

\section{Conclusions}

Our study indicates that the effective clearance rate of aggerates is highest, followed by DC sign, and then tophus. To control the disease effectively, different intensities of ULT should be chosen according to the different MSU burdens detected by US, which can objectively reflect urate crystal 
1 dissolution and provide reliable imaging evidence under efficient ULT. In

2 addition, our results provide a rational basis for the sensitivity of PDUS in

3 detecting the subclinical activity of gout. Although inflammation was

4 gradually reduced after 6 months of treatment, complete control needed for

5 the complete dissolution of MSU deposits. Finally, bone erosions observed

6 on US were stable during the 1-year follow-up period; therefore, early

7 diagnosis and treatment would suggest that US may provide a strong

8 imaging basis for the treatment timing. In summary, US plays an important

9 role in assessing three outcome domain changes in response to effective

10 ULT in patients with established gout.

\section{Abbreviations}

MTP1: First metatarsophalangeal joint; DCS: Double contour sign; ULT: Urate-lowering therapy; ACR: American College of Rheumatology; EULAR: European League Against Rheumatism; GFR: Glomerular filtration rate; BMI: Body mass index; TJC: Tender joint count; SJC: Swollen joint count; OMERACT: Outcome Measures in Rheumatology; T2T: treat to target; GLOESS: Global OMERACT-EULAR Synovitis Score; PDC: Proportion of days covered; BSR: British Society for Rheumatology; MSU: Monosodium urate: NSAID: Nonsteroidal antiinflammatory drug 


\section{Declarations}

\section{Ethics approval and consent to participate}

3 The study was approved by the Ethics Committee of Nanjing Drum Tower

4 Hospital (No.2018-013-01) and all the enrolled patients signed the

5 informed consent form (Trial registration number: ChiCTR1800015043).

6 Consent for publication

$7 \quad$ Not applicable.

$8 \quad$ Availability of data and materials

9 The datasets used and analyzed during the current study are available from

10 the corresponding author on reasonable request.

11 Competing interests

12 The authors declare that they have no competing interests.

$13 \quad$ Funding

14 This work was supported by Nanjing Health and Family Planning 15 Commission through the foundation of Nanjing Science and Technology Development Project for Medicine [grant number YKK18080] to Zhibin

17 Jin.

18 Authors' contributions

19 WZ wrote the initial manuscript; ZJ and HZ was responsible for the study 20 design; WZ and DZ made a contribution to the arrangement of follow-up 21 visit; MW and DZ were responsible for data acquisition and analyses. All 22 the authors have been contributed in revising the manuscript and approved 
1 the final manuscript.

2 Acknowledgements

3 Not applicable

4 Authors' information

$5{ }^{1}$ Department of Ultrasound Medicine, Nanjing Drum Tower Hospital, The

6 Affiliated Hospital of Nanjing University Medical School, No.321

7 Zhongshan Road,210008, Nanjing, Jiangsu, P.R. China. ${ }^{2}$ Department of

8 Rheumatology and Immunology, Nanjing Drum Tower Hospital, The

9 Affiliated Hospital of Nanjing University Medical School, No.321

1. Dalbeth N, Merriman TR, Stamp LK. Gout. Lancet 2016, 388(10055): 2039-2052.

2. Perez-Ruiz F, Moreno-Lledo A, Urionaguena I, Dickson AJ. Treat to target in gout. Rheumatology (Oxford) 2018, 57(suppl_1): i20-i26.

3. Richette P, Doherty M, Pascual E, Barskova V, Becce F, CastanedaSanabria J, et al. 2016 updated EULAR evidence-based recommendations for the management of gout. Ann Rheum Dis 2017, 76(1): 29-42.

4. Khanna D, Fitzgerald JD, Khanna PP, Bae S, Singh MK, Neogi T, et al. 2012 American College of Rheumatology guidelines for 
management of gout. Part 1: Systematic nonpharmacologic and pharmacologic therapeutic approaches to hyperuricemia. Arthritis Care \& Research 2012, 64(10): 1431-1446.

5. Pascual E, Sivera F. Time required for disappearance of urate crystals from synovial fluid after successful hypouricaemic treatment relates to the duration of gout. Ann Rheum Dis 2007, 66(8): 1056-1058.

6. de Lautour H, Taylor WJ, Adebajo A, Alten R, Burgos-Vargas R, Chapman P, et al. Development of Preliminary Remission Criteria for Gout Using Delphi and 1000Minds Consensus Exercises. Arthritis Care \& Research 2016, 68(5): 667-672.

7. Grainger R, Dalbeth N, Keen H, Durcan L, Edwards NL, Perez-Ruiz F, et al. Imaging as an Outcome Measure in Gout Studies: Report from the OMERACT Gout Working Group. Journal Of Rheumatology 2015, 42(12): 2460-2464.

8. Dalbeth N, Doyle AJ. Imaging tools to measure treatment response in gout. Rheumatology (Oxford) 2018, 57(suppl_1): i27-i34.

9. Choi HK, Al-Arfaj AM, Eftekhari A, Munk PL, Shojania K, Reid G, et al. Dual energy computed tomography in tophaceous gout. Ann Rheum Dis 2009, 68(10): 1609-1612.

10. Dalbeth N, Aati O, Kalluru R, Gamble GD, Mcqueen FMJAotRD. Relationship between structural joint damage and urate deposition 
2 11. Ogdie A, Taylor WJ, Neogi T, Fransen J, Jansen TL, Schumacher

in gout: A plain radiography and dual-energy CT study. 2014, 74(6). HR, et al. Performance of Ultrasound in the Diagnosis of Gout in a Multicenter Study Comparison With Monosodium Urate Monohydrate Crystal Analysis as the Gold Standard. Arthritis \& Rheumatology 2017, 69(2): 429-438.

12. Thiele RG, Schlesinger N. Ultrasonography shows disappearance of monosodium urate crystal deposition on hyaline cartilage after sustained normouricemia is achieved. Rheumatol Int 2010, 30(4): 495-503.

13. Peiteado D, Villalba A, Martin-Mola E, de Miguel E. Reduction but not disappearance of Doppler signal after two years of treatment for gout. Do we need a more intensive treatment? Clinical and Experimental Rheumatology 2015, 33(3): 385-390.

14. Ottaviani S, Gill G, Aubrun A, Palazzo E, Meyer O, Dieude P. Ultrasound in gout: A useful tool for following urate-lowering therapy. Joint Bone Spine 2015, 82(1): 42-44.

15. Ebstein E, Forien M, Norkuviene E, Richette P, Mouterde G, Daien C, et al. Ultrasound evaluation in follow-up of urate-lowering therapy in gout: the USEFUL study. Rheumatology (Oxford) 2019, 58(3): 410-417.

16. Scheepers L, Van MO, Stehouwer C, Singh JA, Arts I, Boonen 
AJSiA, et al. Medication adherence among patients with gout: A systematic review and meta-analysis. 2017, 47(5): S0049017217303190.

17. Martinoli C. Musculoskeletal ultrasound: technical guidelines. Insights into Imaging 2010, 1(3): 99-141.

18. Gutierrez M, Schmidt WA, Thiele RG, Keen HI, Kaeley GS, Naredo E, et al. International Consensus for ultrasound lesions in gout: results of Delphi process and web-reliability exercise. Rheumatology 2015, 54(10): 1797-1805.

19. Zhang $\mathrm{W}$, Jin $\mathrm{Z}$, Xiang $\mathrm{W}$, Wu M, Wang $\mathrm{S}$, Zhang $\mathrm{H}$, et al. Ultrasonographic Features of Lower-Limb Joints in Gout: Which Joints and Clinical Characteristics Would Provide More Information for Diagnosis? J Clin Rheumatol 2020, 26(1): 14-18.

20. Szkudlarek M, Court-Payen M, Jacobsen S, Klarlund M, Thomsen HS, Ostergaard M. Interobserver agreement in ultrasonography of the finger and toe joints in rheumatoid arthritis. Arthritis and Rheumatism 2003, 48(4): 955-962.

21. Wakefield RJ, D'Agostino MA, Iagnocco A, Filippucci E, Backhaus M, Scheel AK, et al. The OMERACT Ultrasound Group: status of current activities and research directions. J Rheumatol 2007, 34(4): 848-851.

22. D'Agostino MA, Wakefield RJ, Berner-Hammer H, Vittecoq O, 
Filippou G, Balint P, et al. Value of ultrasonography as a marker of early response to abatacept in patients with rheumatoid arthritis and an inadequate response to methotrexate: results from the APPRAISE study. Ann Rheum Dis 2016, 75(10): 1763-1769.

23. Doherty M, Jansen TL, Nuki G, Pascual E, Perez-Ruiz F, Punzi L, et al. Gout: why is this curable disease so seldom cured? Ann Rheum Dis 2012, 71(11): 1765-1770.

24. Lee YH, Song GG. Diagnostic accuracy of ultrasound in patients with gout: A meta-analysis. Semin Arthritis Rheum 2018, 47(5): 703-709.

25. Modjinou DV, Krasnokutsky S, Gyftopoulos S, Pike VC, Karis E, Keenan RT, et al. Comparison of dual-energy CT, ultrasound and surface measurement for assessing tophi dissolution during rapid urate debulking. Clin Rheumatol 2017, 36(9): 2101-2107.

26. Martillo MA, Nazzal L, Crittenden DB. The crystallization of monosodium urate. Curr Rheumatol Rep 2014, 16(2): 400.

27. Perez-Ruiz F, Calabozo M, Pijoan JI, Herrero-Beites AM, Ruibal A. Effect of urate-lowering therapy on the velocity of size reduction of tophi in chronic gout. Arthritis Rheum 2002, 47(4): 356-360.

28. Perez-Ruiz F, Martin I, Canteli B. Ultrasonographic measurement of tophi as an outcome measure for chronic gout. J Rheumatol 2007, 34(9): 1888-1893. 
1 29. Das S, Goswami RP, Ghosh A, Ghosh P, Lahiri D, Basu K. Temporal evolution of urate crystal deposition over articular cartilage after successful urate-lowering therapy in patients with gout: An ultrasonographic perspective. Modern Rheumatology 2017, 27(3): 518-523.

30. Peiteado D, Villalba A, Martin-Mola E, Balsa A, De Miguel E. Ultrasound sensitivity to changes in gout: a longitudinal study after two years of treatment. Clin Exp Rheumatol 2017, 35(5): 746-751.

31. Dalbeth N, Pool B, Gamble GD, Smith T, Callon KE, McQueen FM, et al. Cellular characterization of the gouty tophi: a quantitative analysis. Arthritis Rheum 2010, 62(5): 1549-1556.

32. Ten Cate DF, Luime JJ, Swen N, Gerards AH, De Jager MH, Basoski $\mathrm{NM}$, et al. Role of ultrasonography in diagnosing early rheumatoid arthritis and remission of rheumatoid arthritis--a systematic review of the literature. Arthritis Res Ther 2013, 15(1): R4.

33. Gutierrez M, Di Geso L, Salaffi F, Bertolazzi C, Tardella M, Filosa G, et al. Development of a preliminary US power Doppler composite score for monitoring treatment in PsA. Rheumatology (Oxford) 2012, 51(7): 1261-1268.

34. Ficjan A, Husic R, Gretler J, Lackner A, Graninger WB, Gutierrez $\mathrm{M}$, et al. Ultrasound composite scores for the assessment of inflammatory and structural pathologies in Psoriatic Arthritis 
(PsASon-Score). Arthritis Res Ther 2014, 16(5): 476.

35. Dalbeth N, Saag KG, Palmer WE, Choi HK, Hunt B, MacDonald PA, et al. Effects of Febuxostat in Early Gout: A Randomized, Double-Blind, Placebo-Controlled Study. Arthritis Rheumatol 2017, 69(12): 2386-2395.

36. Shoji A, Yamanaka H, Rheum NKJA. A retrospective study of the relationship between serum urate level and recurrent attacks of gouty arthritis: Evidence for reduction of recurrent gouty arthritis with antihyperuricemic therapy. Arthritis Rheum 2004, 51(3): 321-325.

37. Schueller-Weidekamm C, Schueller G, Aringer M, Weber M, Kainberger F. Impact of sonography in gouty arthritis: comparison with conventional radiography, clinical examination, and laboratory findings. Eur J Radiol 2007, 62(3): 437-443.

38. Cazenave T, Martire V, Reginato AM, Gutierrez M, Waimann CA, Pineda C, et al. Reliability of OMERACT ultrasound elementary lesions in gout: results from a multicenter exercise. Rheumatol Int 2019, 39(4): 707-713.

39. Eason A, House ME, Vincent Z, Knight J, Tan P, Horne A, et al. Factors associated with change in radiographic damage scores in gout: a prospective observational study. Ann Rheum Dis 2016, 75(12): 2075-2079.

40. Dalbeth N, Clark B, Gregory K, Gamble G, Sheehan T, Doyle A, et 
al. Mechanisms of bone erosion in gout: a quantitative analysis using plain radiography and computed tomography. Ann Rheum Dis 2009, 68(8): 1290-1295.

41. Wu M, Liu FJ, Jiang LX, Chen J, Chen L, Wei C, et al. The prevalence and factors associated with bone erosion in patients with gout. Arthritis Care Res (Hoboken) 2018.

42. Dalbeth N, Smith T, Nicolson B, Clark B, Callon K, Naot D, et al. Enhanced osteoclastogenesis in patients with tophaceous gout: urate crystals promote osteoclast development through interactions with stromal cells. Arthritis Rheum 2008, 58(6): 1854-1865.

43. Chhana A, Callon KE, Pool B, Naot D, Watson M, Gamble GD, et al. Monosodium urate monohydrate crystals inhibit osteoblast viability and function: implications for development of bone erosion in gout. Ann Rheum Dis 2011, 70(9): 1684-1691.

44. Dalbeth N, Doyle AJ, McQueen FM, Sundy J, Baraf HS. Exploratory study of radiographic change in patients with tophaceous gout treated with intensive urate-lowering therapy. Arthritis Care Res (Hoboken) 2014, 66(1): 82-85.

45. Dalbeth N, Aati O, Gamble GD, Horne A, House ME, Roger M, et al. Zoledronate for prevention of bone erosion in tophaceous gout: a randomised, double-blind, placebo-controlled trial. Ann Rheum Dis 2014, 73(6): 1044-1051. 
1 46. Garner HW, Wessell DE. Current status of ultrasound and dualenergy computed tomography in the evaluation of gout. Rheumatol Int 2018, 38(8): 1339-1344.

47. Yu KH, Chen DY, Chen JH, Chen SY, Chen SM, Cheng TT, et al. Management of gout and hyperuricemia: Multidisciplinary consensus in Taiwan. Int J Rheum Dis 2018, 21(4): 772-787. 

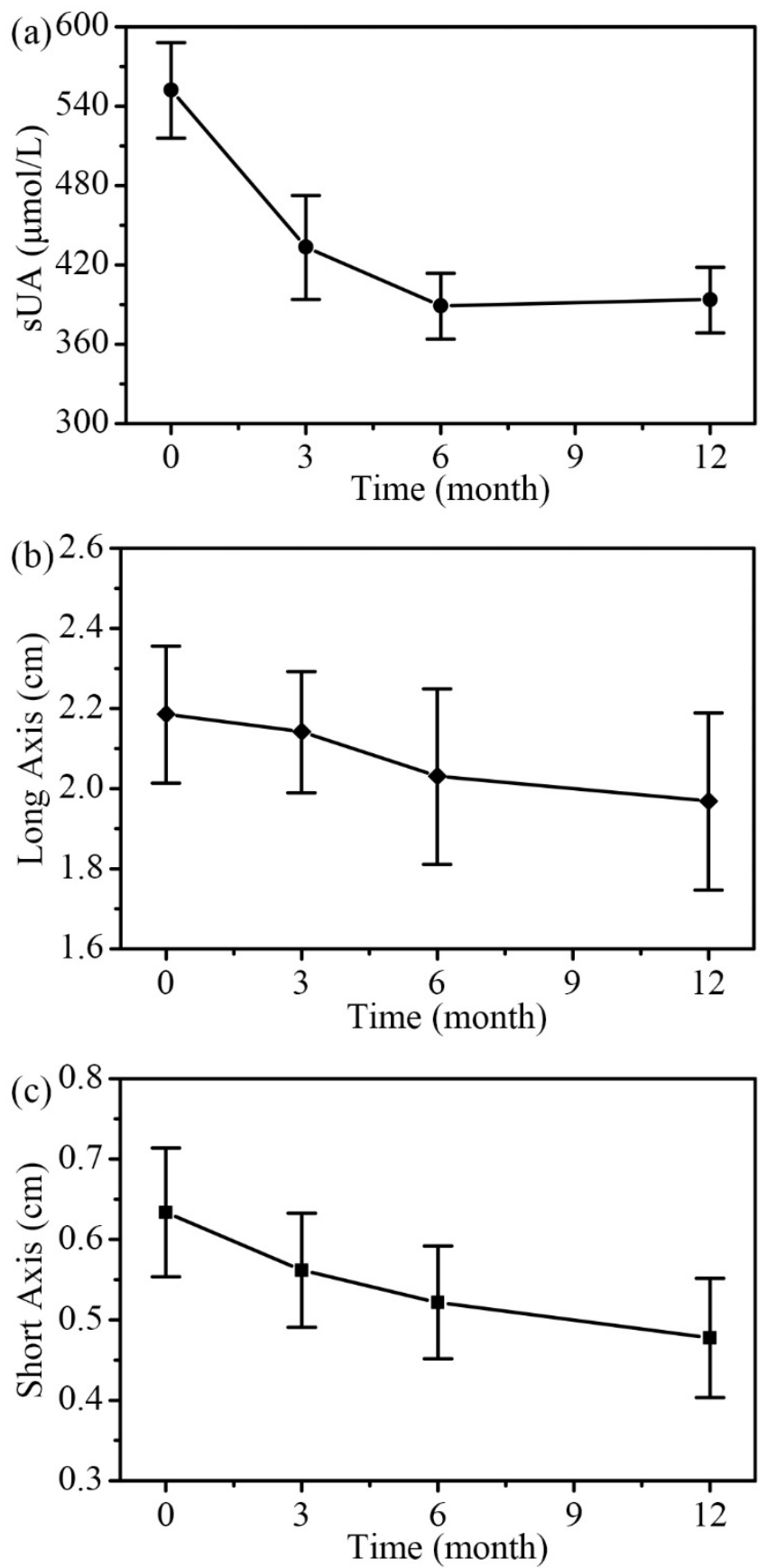

Fig 1. The decline trend of serum urate level (a), long axis (b) and short axis (c) diameter of tophi 4 after receiving 3, 6 and 12 months of urate-lowering treatment. 

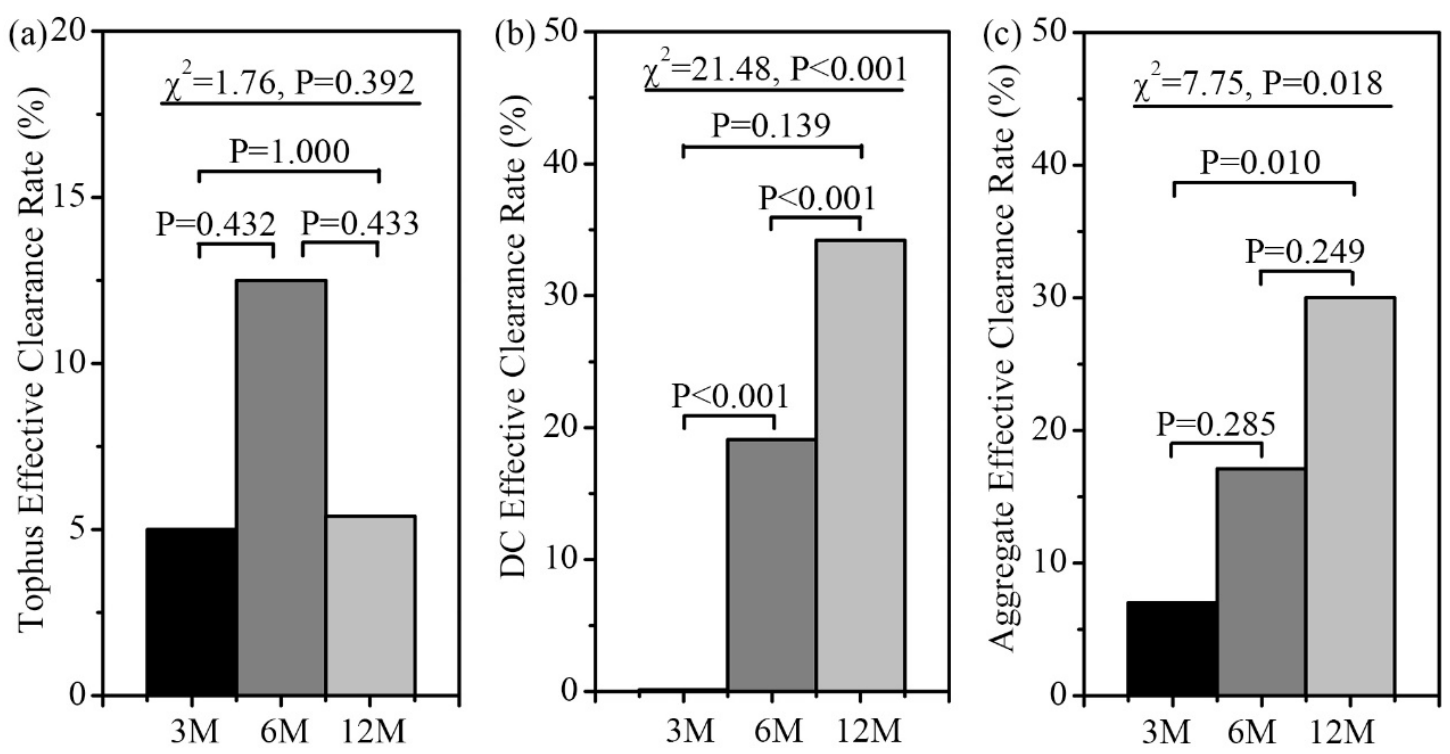

2 Fig. 2 Comparison of effective clearance rates of tophi, DC and aggregates between different time 3 period

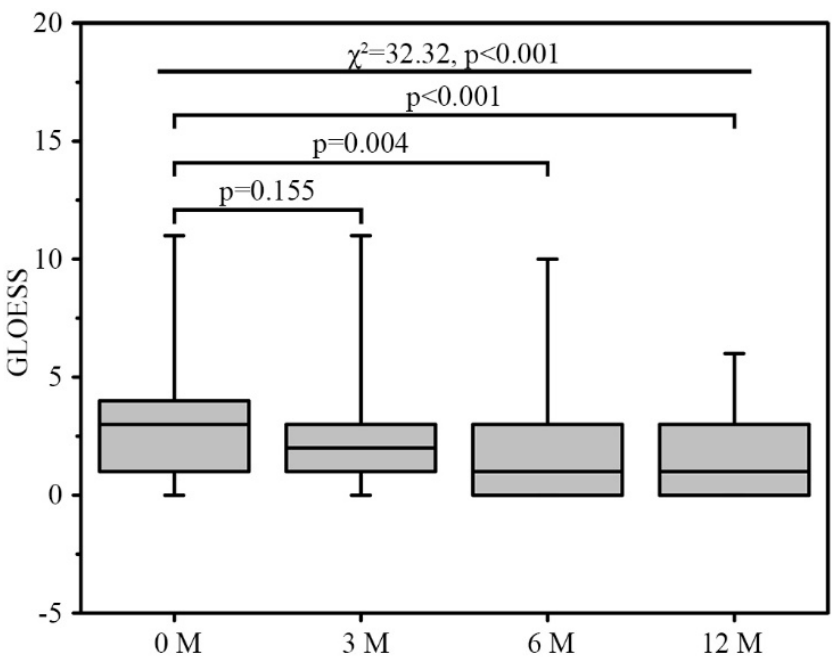

Fig. 3 Comparison among groups of GLOESS between different time period

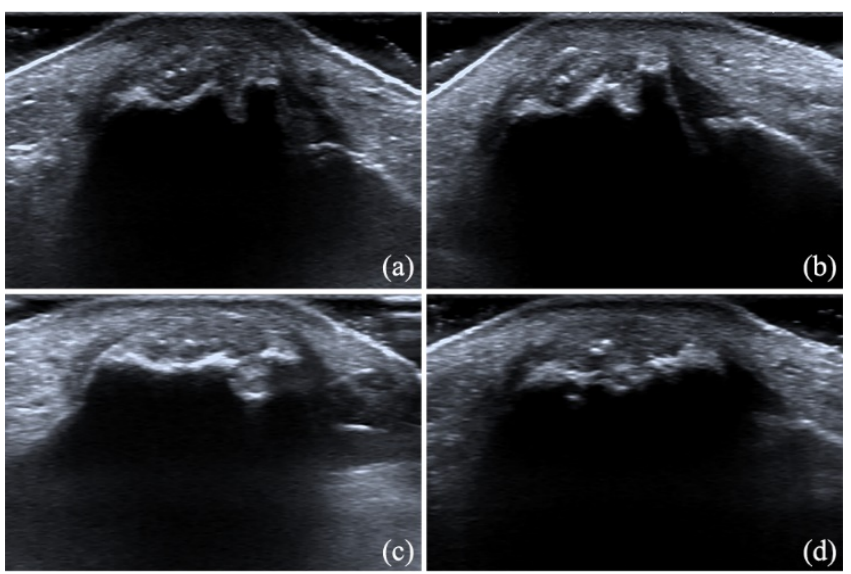

Fig. 4 lateral longitudinal view of the first metatarsophalangeal joint. There was no significant change in bone erosion at baseline period (a), 3 (b), 6 (c) and12 months (d) that the scores were all 
graded 3.

\section{Tables}

Table1 Baseline demographics and clinical characteristics

\begin{tabular}{|c|c|c|c|c|}
\hline & Baseline & Month 3 & Month 6 & Month 12 \\
\hline Family history & $36(45.6)$ & / & / & / \\
\hline High purine diet & $45(57)$ & I & I & I \\
\hline Drinking history & $47(59.5)$ & l & I & I \\
\hline Hyperlipidemia & $22(27.8)$ & I & I & I \\
\hline Hypertension & $29(36.7)$ & I & I & I \\
\hline Diabetes & $7(8.9)$ & l & / & I \\
\hline $\begin{array}{l}\text { Coronary heart } \\
\text { disease }\end{array}$ & $3(3.8)$ & l & I & I \\
\hline $\begin{array}{l}\mathrm{BMI} \\
\left(\bar{x} \pm \mathrm{s}, \mathrm{kg} / \mathrm{m}^{2}\right)\end{array}$ & $26.5 \pm 2.9$ & I & l & I \\
\hline SJC(M/QR) & $1(0,2)$ & $0(0,1)$ & $0(0,0) \boldsymbol{\Delta}$ & $0(0,0) \&$ \\
\hline TJC(M/QR) & $1(1,2)$ & $0(0,1) \&$ & $0(0,1) \&$ & $0(0,0) *$ \\
\hline $\begin{array}{l}\text { Frequency of } \\
\text { attacks } \\
\text { (number/year, } \\
\mathrm{M} / \mathrm{QR} \text { ) }\end{array}$ & $5(2,15)$ & $4(0,12)$ & $4(0,11)$ & $0(0,4) * \#$ \\
\hline $\begin{array}{l}\text { Duration time } \\
\text { (day, M/QR) }\end{array}$ & $7(3,7)$ & $2(0,5) \&$ & $2(0,3) \&$ & $0(0,2) *$ \\
\hline $\begin{array}{l}\text { sUA level } \\
(\bar{x} \pm \mathrm{s}, \mu \mathrm{mol} / \mathrm{L})\end{array}$ & $552.1 \pm 103.4$ & $433.3 \pm 112.5^{*}$ & $388.9 \pm 71.1^{*}$ & $393.5 \pm 71.2 *$ \\
\hline $\begin{array}{l}\text { BUN } \\
(\bar{x} \pm \mathrm{s}, \mathrm{mmol} / \mathrm{L})\end{array}$ & $5.4 \pm 1.9$ & $5.2 \pm 1.3$ & $5.6 \pm 1.4$ & $5.6 \pm 1.8$ \\
\hline $\begin{array}{l}\text { Scr } \\
(\bar{x} \pm \mathrm{s}, \mu \mathrm{mol} / \mathrm{L})\end{array}$ & $90.7 \pm 23.2$ & $85.3 \pm 27.9$ & $84.3 \pm 22.1$ & $90.2 \pm 28.0$ \\
\hline
\end{tabular}

Data are number (\%) unless indicated. BMI, body mass index; SJC, swollen joint count; TJC, tender joint count; M: median; QR: interquartile range; sUA, serum uric acid; BUN, blood urea nitrogen; Scr, serum creatinine*,compared with baseline data, $\mathrm{P} \leqslant 0.001 ; \quad \&$,compared with baseline data, $\mathrm{P} \leqslant 0.01 ; \#$, compared with month $3, \mathrm{P}<0.01 ; \boldsymbol{\Delta}$, compared with baseline data, $\mathrm{P}<0.05$ 
Table 2 modification of tophi, DC and aggregates during the follow-up process

\begin{tabular}{lcccc}
\hline & $\begin{array}{c}\text { Baseline } \\
\mathrm{N}=79\end{array}$ & $\begin{array}{r}\text { Month 3 } \\
\mathrm{N}=67\end{array}$ & $\begin{array}{r}\text { Month 6 } \\
\mathrm{N}=64\end{array}$ & $\begin{array}{c}\text { Month 12 } \\
\mathrm{N}=57\end{array}$ \\
\hline $\begin{array}{l}\text { Tophi, } \mathrm{n}(\%) \\
\text { Long axis diameter of tophi }\end{array}$ & $48(60.8)$ & $40(59.7)$ & $38(59.4)$ & $35(61.4)$ \\
$(\bar{x} \pm \mathrm{s}, \mathrm{cm})$ & $2.2 \pm 0.43$ & $2.1 \pm 0.38$ & $2.0 \pm 0.55$ & $1.9 \pm 0.56$ \\
$\begin{array}{l}\text { Short axis diameter of tophi } \\
(\bar{x} \pm \mathrm{s}, \mathrm{cm})\end{array}$ & $0.63 \pm 0.20$ & $0.56 \pm 0.18$ & $0.52 \pm 0.18$ & $0.48 \pm 0.19$ \\
DC, $\mathrm{n}(\%)$ & $53(67.1)$ & $48(71.6)$ & $38(59.4)$ & $19(33.3)$ \\
Aggregates, $\mathrm{n}(\%)$ & $44(55.7)$ & $39(58.2)$ & $29(45.3)$ & $17(29.8)$ \\
\hline
\end{tabular}


Figures
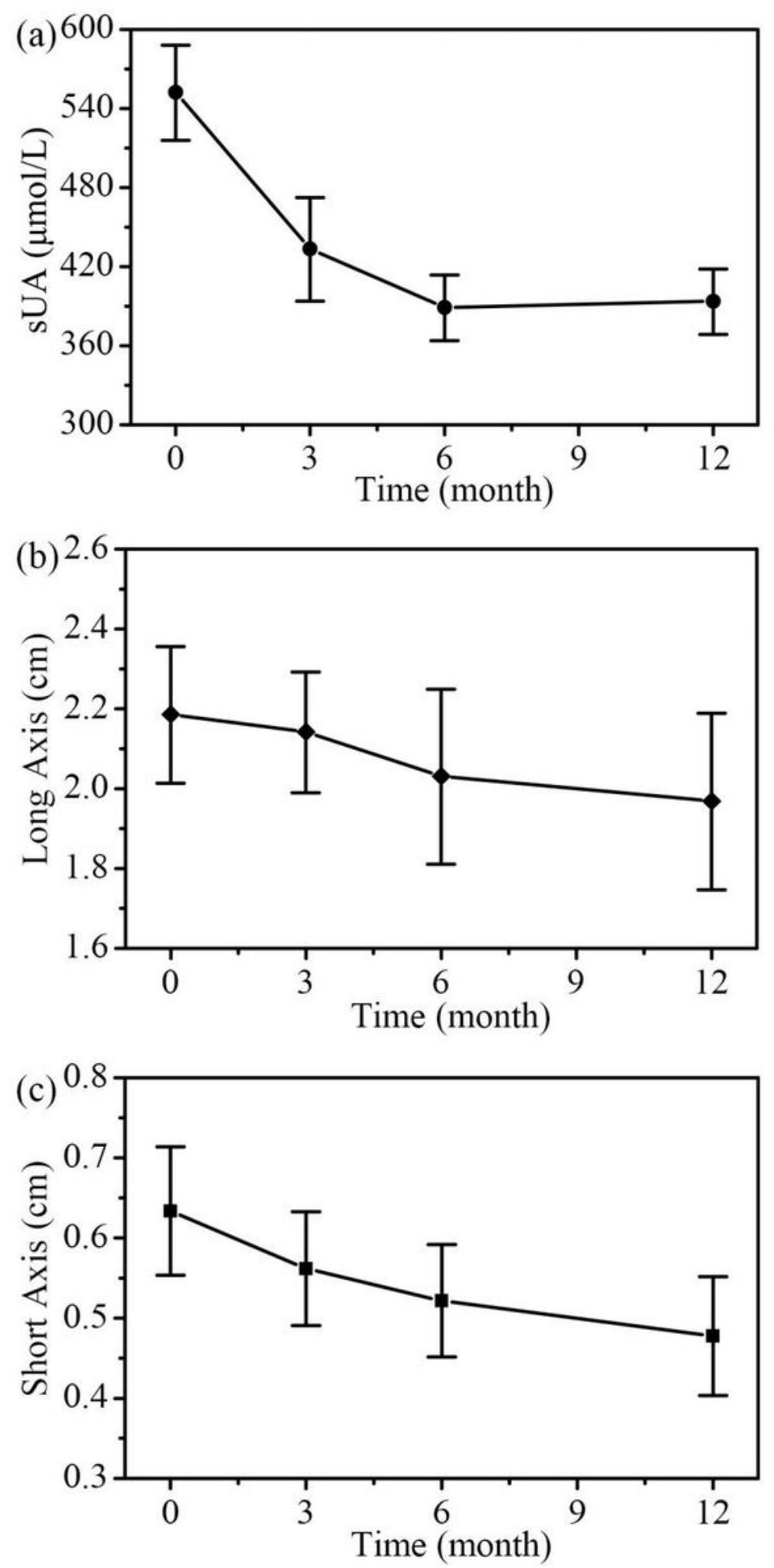

\section{Figure 1}

The decline trend of serum urate level (a), long axis (b) and short axis (c) diameter of tophi after receiving 3,6 and 12 months of urate-lowering treatment. 

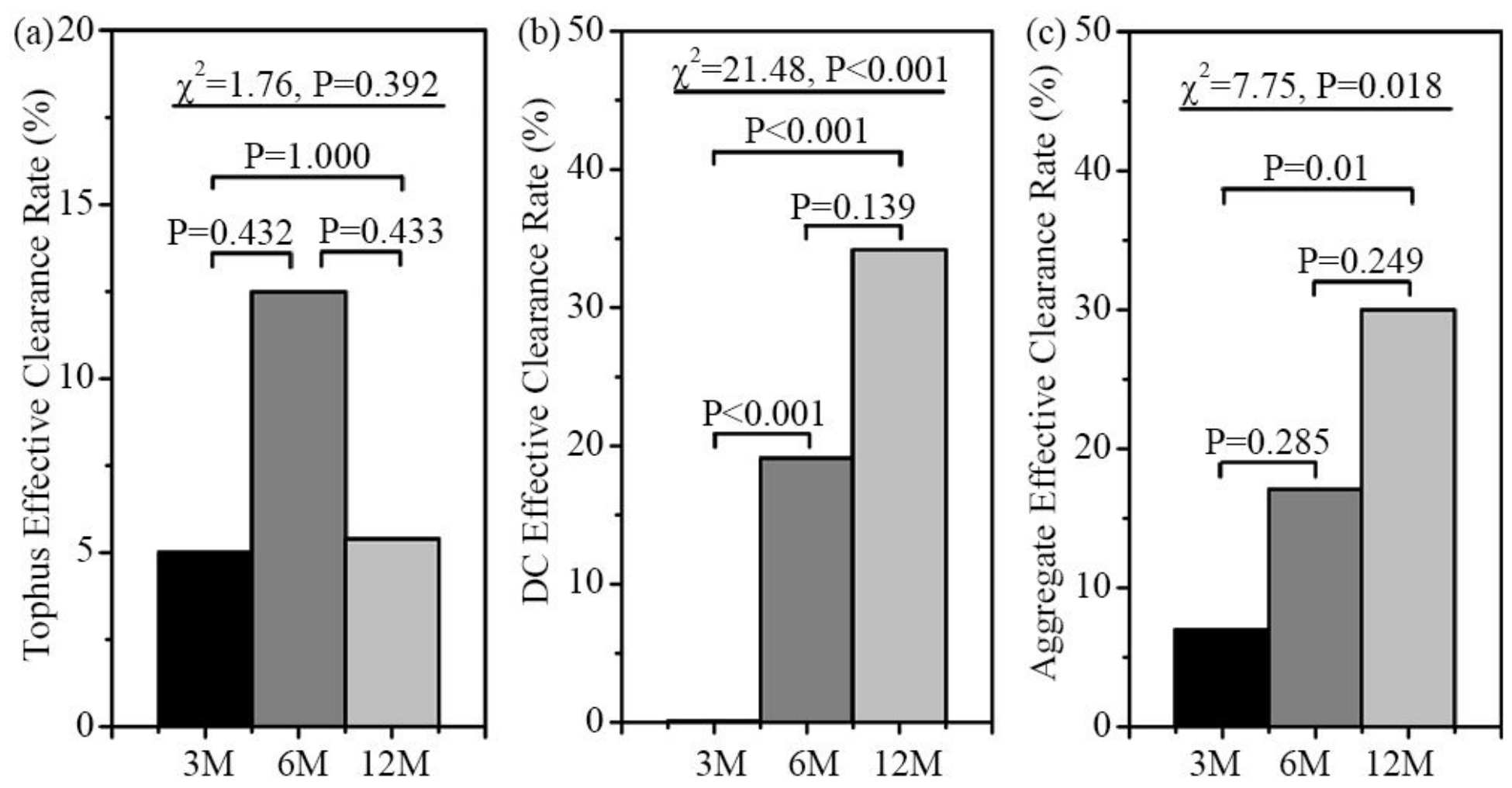

Figure 2

Comparison of effective clearance rates of tophi, DC and aggregates between different time period 


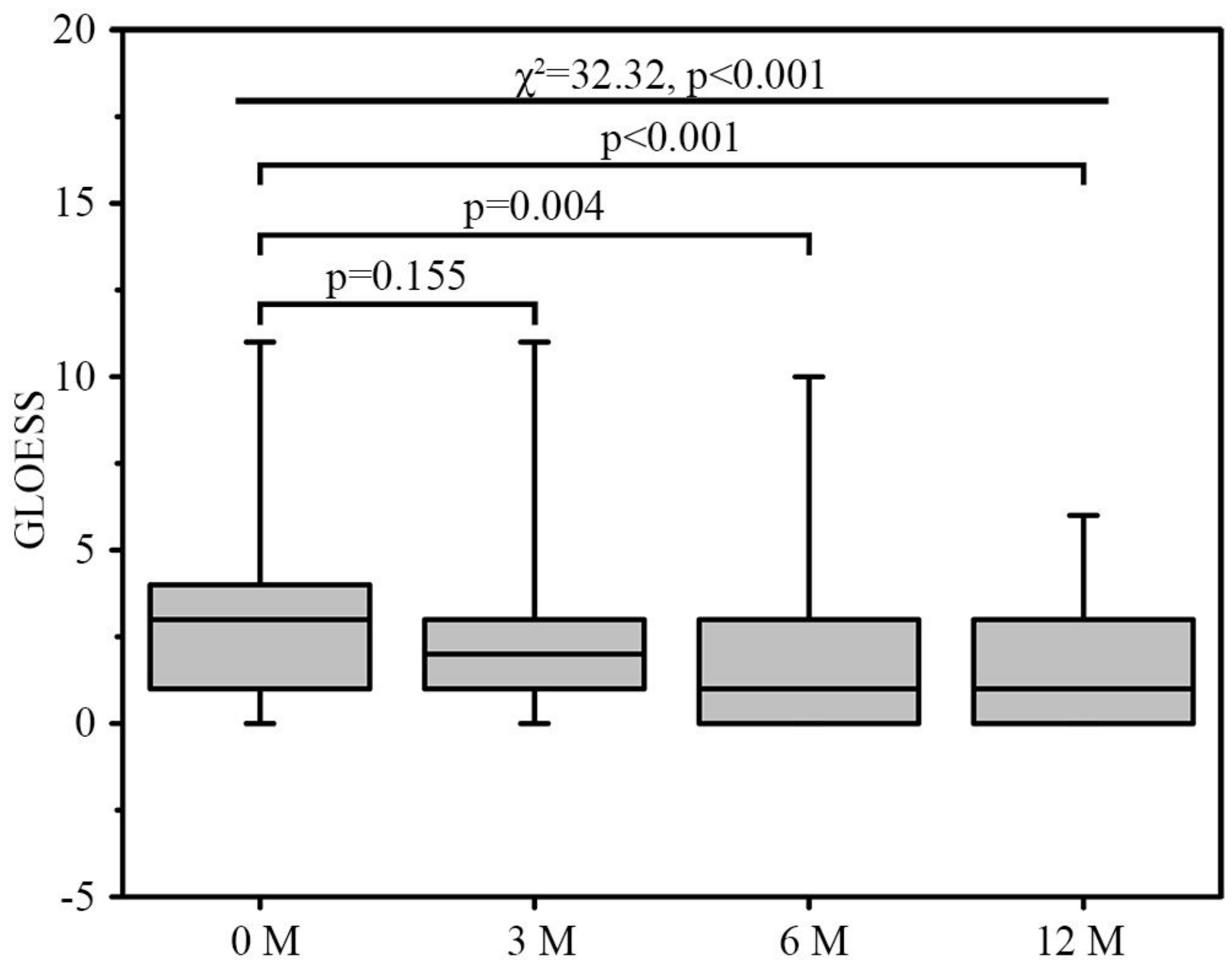

Figure 3

Comparison among groups of GLOESS between different time period 

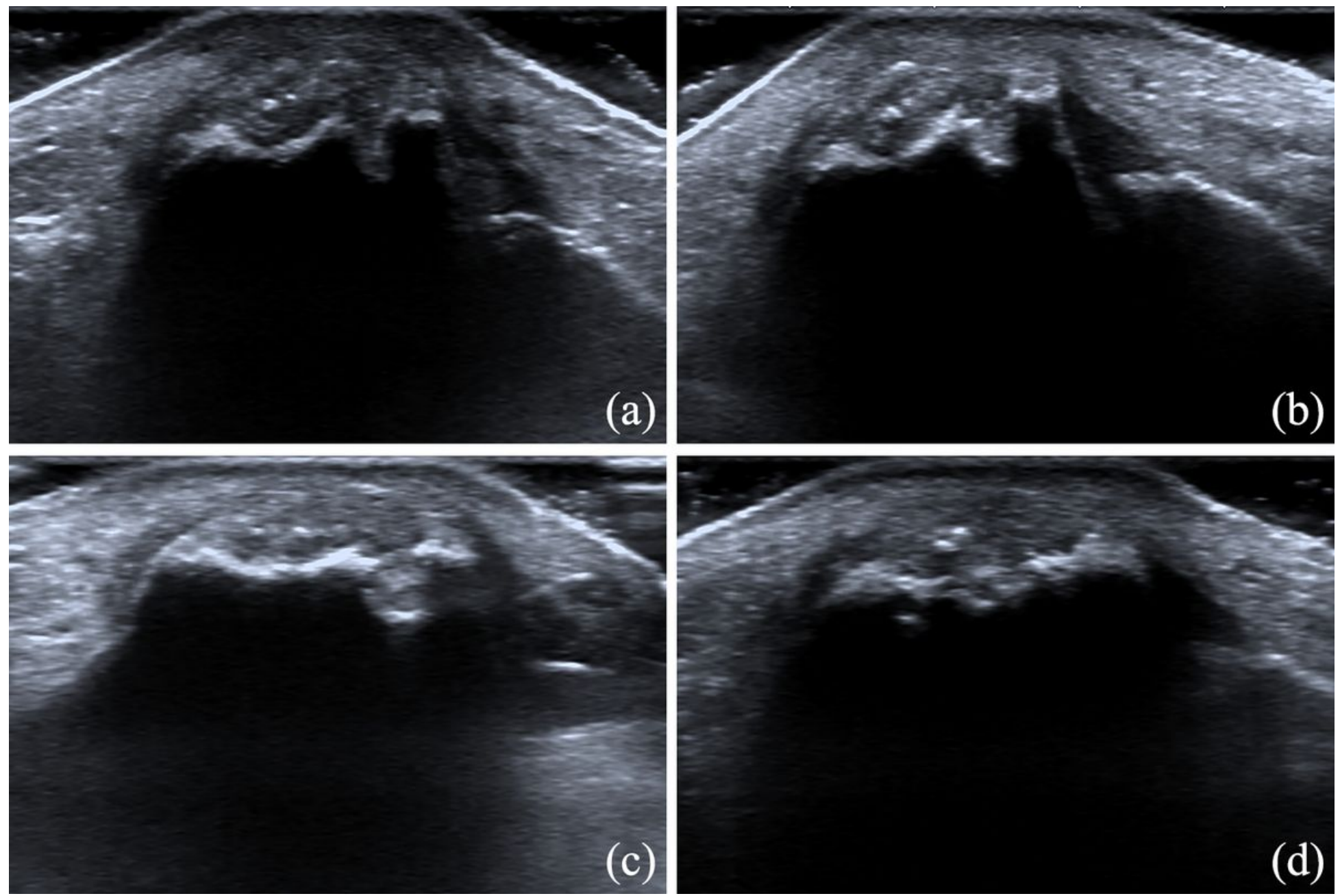

Figure 4

lateral longitudinal view of the first metatarsophalangeal joint. There was no significant change in bone erosion at baseline period (a), 3 (b), 6 (c) and12 months (d) that the scores were all graded 3. 